\title{
The RNA binding protein hnRNPK protects against adriamycin- induced podocyte injury
}

\author{
Shili Zhao ${ }^{1,2}$, Junxia Feng ${ }^{2}$, Jingchun $\mathrm{Li}^{2}$, Rui Cao ${ }^{1}$, Yunfang Zhang ${ }^{2}$, Shen Yang ${ }^{2}$, Lianghong Yin ${ }^{1}$ \\ ${ }^{1}$ Department of Nephrology, The First Affiliated Hospital of Jinan University, Guangzhou, China; ${ }^{2}$ Department of Nephrology, Affiliated Huadu \\ Hospital, (People's Hospital of Huadu District) The Third School of Clinical Medicine, Southern Medical University, Guangzhou, China \\ Contributions: (I) Conception and design: S Zhao, S Yang, L Yin; (II) Administrative support: L Yin, S Zhao; (III) Provision of study materials or \\ patients: S Zhao, J Feng; (IV) Collection and assembly of data: J Feng, R Cao, J Li; (V) Data analysis and interpretation: S Zhao, J Feng, J Li, Y \\ Zhang; (VI) Manuscript writing: All authors; (VII) Final approval of manuscript: All authors. \\ Correspondence to: Shen Yang. Department of Nephrology, Affiliated Huadu Hospital, Southern Medical University (People's Hospital of Huadu \\ District), No. 48 Xinhua Road, Huadu District, Guangzhou 510800, China. Email: 13424028260@163.com; Lianghong Yin. Department of \\ Nephrology, The First Affiliated Hospital of Jinan University, Guangzhou 510632, China. Email: yin-yun@126.com.
}

Background: Podocytes maintain the integrity of the glomerular filtration barrier and serve as the final barrier to protein loss. Podocyte injury may induce severe apoptosis, which can result in serious kidney damage and disease. Therefore, it is necessary to explore how podocyte injury can be prevented and to thereby discover a feasible therapy for kidney disease. However, the mechanism of podocyte injury is still unclear.

Methods: The mRNA and protein expression levels of synaptopodin and nephrin in MPC5 podocytes with adriamycin (ADR)-induced injury were detected by quantitative real-time PCR and western blot. The expression levels of heterogeneous nucleotide protein K (hnRNPK), caspase-3, Bax, and Bcl-2 protein in cells and tissues were measured using western blot. Proliferation were measured in treated MPC5 podocytes by Cell Counting Kit-8 (CCK-8) assay, EdU assay, and apoptosis was measured by Hoechst 32258 staining. Mitochondrial membrane potential disruption, lactate dehydrogenase (LDH) leakage, and reactive oxygen species (ROS) generation were measured using JC-1 staining, an LDH reagent kit, and a ROS detection kit. Hematoxylin and eosin (HE) staining was used to observe histological changes in mouse tissues.

Results: Synaptopodin and nephrin were downregulated in ADR-treated podocytes. Overexpression of hnRNPK ameliorated the inhibitive effect of ADR treatment on podocyte proliferation and reduced its promotion of podocyte apoptosis. LDH leakage and ROS generation were increased in ADR-treated podocytes, but were reduced by hnRNPK treatment.

Conclusions: ADR-induced podocyte injury is ameliorated by hnRNPK both in vivo and in vitro. This observation provides a basis for a feasible therapy to prevent podocyte injury and subsequent kidney disease.

Keywords: Podocyte injury; adriamycin (ADR); heterogeneous nucleotide protein K (hnRNPK); apoptosis

Submitted May 20, 2021. Accepted for publication Aug 11, 2021.

doi: 10.21037/atm-21-3577

View this article at: https://dx.doi.org/10.21037/atm-21-3577

\section{Introduction}

Podocytes are highly terminally differentiated epithelial cells which attach to the outer layer of the glomerular basement membrane and form a complex cellular structure in the kidney (1). This structure maintains the integrity of the glomerular filtration barrier, in which podocytes filter out water and metabolic waste as blood flows through each glomerulus. Podocytes can therefore be considered as the last barrier to protein loss $(2,3)$. An early sign of podocyte injury is decreased expression of podocyte cytoskeletal proteins, such as synaptopodin, nephrin, and podocin, which leads to cytoskeleton disorders, foot process fusion, 
and proteinuria production. Podocytes are non-renewable, and their sustained injury may cause severe apoptosis, potentially leading to serious kidney damage and disease (4-7). Many studies have explored the prevention of podocyte injury in an effort to find a feasible therapy to treat kidney disease; however, the mechanism of podocyte injury has proved elusive.

Heterogeneous nucleotide protein $\mathrm{K}(\mathrm{hnRNPK})$ is a functional regulatory protein that is widely expressed in a variety of human cells and plays an important role in gene regulation, cancer, and skeletal development $(8,9)$. In recent years, hnRNPK has attracted increasing attention as a tumor suppressor. For instance, a previous study showed that it plays a tumor suppressor role in hematological cancer (10). Furthermore, Abdo et al. reported that hnRNPK was downregulated in diabetic Akita mice and that hnRNPK overexpression could inhibit the transcription of renal AGT, thereby improving the ability of insulin to prevent hypertension and, to a certain extent, reducing renal injury (11). However, the correlation between podocyte injury and hnRNPK has scarcely been studied. Consequently, the present study aimed to explore how hnRNPK impacts podocyte injury.

In this study, we first induced injury in podocytes by treating them with adriamycin (ADR). Podocyte proliferation and apoptosis were examined, and the results showed that ADR treatment caused obvious cell apoptosis. We also found that hnRNPK expression was low in ADRinjured podocytes and that overexpression of hnRNPK could alleviate the effects of injury. This study has provided a basis for a feasible therapy to prevent podocyte injury and subsequent kidney disease. We present the following article in accordance with the ARRIVE reporting checklist (available at https://dx.doi.org/10.21037/atm-21-3577).

\section{Methods}

\section{Establishment of an ADR-induced nephropathy animal model}

Male mice (weight: 18-22 g, age: 6 weeks) were purchased from the Animal Experiment Center of Southern Medical University. All mice were housed under specific pathogenfree (SPF) conditions and allowed free water and food access. All procedures involving animals were performed and monitored in compliance with the guidelines of the Affiliated Huadu Hospital, Southern Medical University Animal Use Regulations and approved by The Institutional Animal
Care and Use Committee (IACUC) of the Affiliated Huadu Hospital, Southern Medical University. An ADR-induced nephropathy animal model was constructed by injecting 10.5 $\mathrm{mg} / \mathrm{kg}$ ADR through the caudal vein of the mice.

The $h n R N P K$ gene $(1,395 \mathrm{bp})$ was amplified using $2 \times$ Phanta Master Mix (Vazyme, Guangzhou, China), and the products were cloned into the pcDNA3.0 vector. The primers were Hnrnpk-F1, 5'-GGGGTACCATGGAGAC CGAACAGCCAGAAG-3' and Hnrnpk-R1 5'-CCGCT CGAGTTAGAATCCTTCAACATCTGCATACTG-3' . The endonucleases utilized were KpnI (Thermo) and XhoI (Thermo), respectively. The successful construction of the hnRNPK overexpression plasmid was confirmed by sequencing. For control, pcDNA3.0 vector was applied.

The mice were randomly divided into 4 groups: control $(\mathrm{n}=5)$, model $(\mathrm{n}=5)$, model + vector $(\mathrm{n}=5)$ and model + hnRNPK $(n=5)$. The model mice were treated with ADR. To evaluate the effects of hnRNPK on kidney injury, the model + vector and model + hnRNPK mice were injected with vector and hnRNPK $(15 \mu \mathrm{g})$, respectively, via the tail vein, at the same time as ADR injection. After 2 weeks, the mice were sacrificed and glomeruli were obtained for further experiments.

\section{Cell culture and treatment}

Immortalized mouse podocytes (MPC5) were purchased from Tong Pai Bio-tech Company (Shanghai, China). After thawing, the podocytes were cultured in Roswell Park Memorial Institute 1640 Medium (Thermo Scientific, Waltham, USA) supplemented with $10 \% \mathrm{v} / \mathrm{v}$ fetal bovine serum (Gibco FBS, NY, USA). Podocytes were then grown in a medium containing $10 \mathrm{U} / \mathrm{mL}$ interferon- $\gamma$ (BioSource, Waltham, USA) in a humid incubator containing $5 \% \mathrm{CO}_{2}$ at $37{ }^{\circ} \mathrm{C}$. The podocytes in the model group were treated with ADR (100 nM). The model podocytes $\left(1 \times 10^{5}\right.$ cells) were seeded in a 6-well plate and transfected with hnRNPKoverexpression plasmid and vector using Lipofectamine 3000 (Invitrogen), in line with the manufacturer's instructions.

\section{$R N A$ extraction and quantitative real-time PCR}

The extraction of total RNA from MPC5 podocytes was performed using TRIzol Reagent (Invitrogen, Carlsbad, CA, USA), after which the RNA quality was detected using the NanoDrop 2000c spectrophotometer (Thermo Scientific, Waltham, USA), in adherence with the manufacturer's protocols. Next, the RNA was converted 
into complementary DNA (cDNA) using the RevertAid First Strand cDNA Synthesis kit (Thermo), and qRT-PCR was performed on an ABI 7900 system using SYBR Green Real-Time PCR master mix (Thermo).

The primer sequences used were: GAPDH, F: 5'-T GT TCGTCATGGGTGTGAAC-3', R: 5'-ATGGCATGG ACTGTGGTCAT-3'; synaptopodin, F:5'-GCTCGAAT TCCGATGCAAATAAAC-3', R:5'-CAGGCCACAGTGA GATGTGAAGA-3'; nephrin, F:5'-CAGGGAAGACAG CAACAAACAA-3', R: 5'-CAGGTTTTCAGATAGA GCCCAGA-3'. All primers were synthesized by RiboBio (Guangzhou, China).

\section{Western blot}

Total protein was extracted using cell lysate. After quantification of the protein concentration using the Bradford Protein Assay Kit (Beyotime Institute of Biotechnology, Haimen, China), the samples were separated by $10 \%$ sodium dodecyl sulfate-polyacrylamide gel electrophoresis, and then transferred to polyvinylidene difluoride membranes (Millipore, MA, USA) and blocked with $5 \%$ skim milk for 1 hour at room temperature. Subsequently, the membranes were subjected to incubation with primary antibodies against synaptopodin $(1: 1,000$, ab224491, Abcam), nephrin (1:1,000, ab21634, Abcam), caspase-3 (1:2,000, ab184787, Abcam), Bax (1:5,000, ab32503, Abcam), Bcl-2 (1:500, ab59348, Abcam), and hnRNPK $\left(1: 1,000\right.$, ab39975, Abcam) overnight at $4{ }^{\circ} \mathrm{C}$, and then with the corresponding secondary antibodies at $37^{\circ} \mathrm{C}$ for 1 hour. Finally, the protein bands were visualized using ECL reagent (Millipore, Bedford, MA, USA), with GAPDH acting as an internal control.

\section{Cell Counting Kit-8 (CCK-8) assay}

The effects of ADR and hnRNPK on the cell viability of MPC5 podocytes were evaluated using the CCK-8) assay (Beyotime). After being plated on 96-well plates overnight at a density of 5,000 cells/well, podocytes were transfected with $\mathrm{ADR}$ or $\mathrm{ADR}+\mathrm{hnRNPK}$. After incubation for 0,24 , or 48 hours, $10 \mu \mathrm{L}$ of CCK- 8 solution was added to each well and the podocytes were then incubated for 1 hour at $37^{\circ} \mathrm{C}$. Next, the transfected cells were detected at $450 \mathrm{~nm}$ using a spectrophotometer. The results represent the mean of three replicates under identical conditions.

\section{EdU assay}

Podocyte proliferation was measured using the EdU assay kit (Riobio, Guangzhou, China). Podocytes were treated with ADR or ADR + hnRNPK, or their negative controls for 24 hours. After two washes with phosphate-buffered saline (PBS), treated podocytes were fixed with $0.5 \%$ buffered paraformaldehyde, and then incubated with the EdU detection solution for 30 minutes in the dark. Finally, a flow cytometer (Becton Dickinson, USA) was used to detect cell proliferation.

\section{Hoechst 32258 staining}

Podocytes were seeded into 6-well plates $\left(10^{4}\right.$ cells/well $)$, after which they were fixed with $4 \%$ paraformaldehyde for 15 minutes and washed with PBS. Hoechst 33258 (Sigma-Aldrich) was applied to the podocytes, which were incubated in the dark for 10 minutes. Apoptotic nuclei were analyzed by fluorescence microscopy (Olympus, Japan).

\section{Mitochondrial membrane potential assay ( $\mathcal{F C}-1$ assay)}

The mitochondrial membrane potential (MMP, $\Delta \Psi \mathrm{m}$ ) of MPC5 podocytes was assessed using the JC-1 staining kit (Beyotime), following the standard kit instructions. Podocytes were washed twice with PBS and plated in a 6 -well plate. JC-1 dye was added to each well, and the podocytes were subsequently incubated in the dark at $37^{\circ} \mathrm{C}$ for 20 minutes. After washing with cold JC-1 staining buffer, cultured podocytes were quantified by flow cytometry to detect the fluorescent cells.

\section{Lactate debydrogenase $(\mathrm{LDH})$ release assay}

To analyze cell damage, LDH release was quantified based on the enzymatic reaction. Podocytes were seeded into a 96-well plate and cultured for 24 hours under different treatments. Then, the supernatant was collected, and the $\mathrm{LDH}$ level was measured at $450 \mathrm{~nm}$ with a microplate reader using a reagent kit (Nanjing Jiancheng Biology Engineering Institute, China). Finally, the percentage of $\mathrm{LDH}$ release was quantified.

\section{Intracellular reactive oxygen species (ROS) detection}

Podocytes were incubated with $2^{\prime}, 7^{\prime}$-dichlorofluorescin 

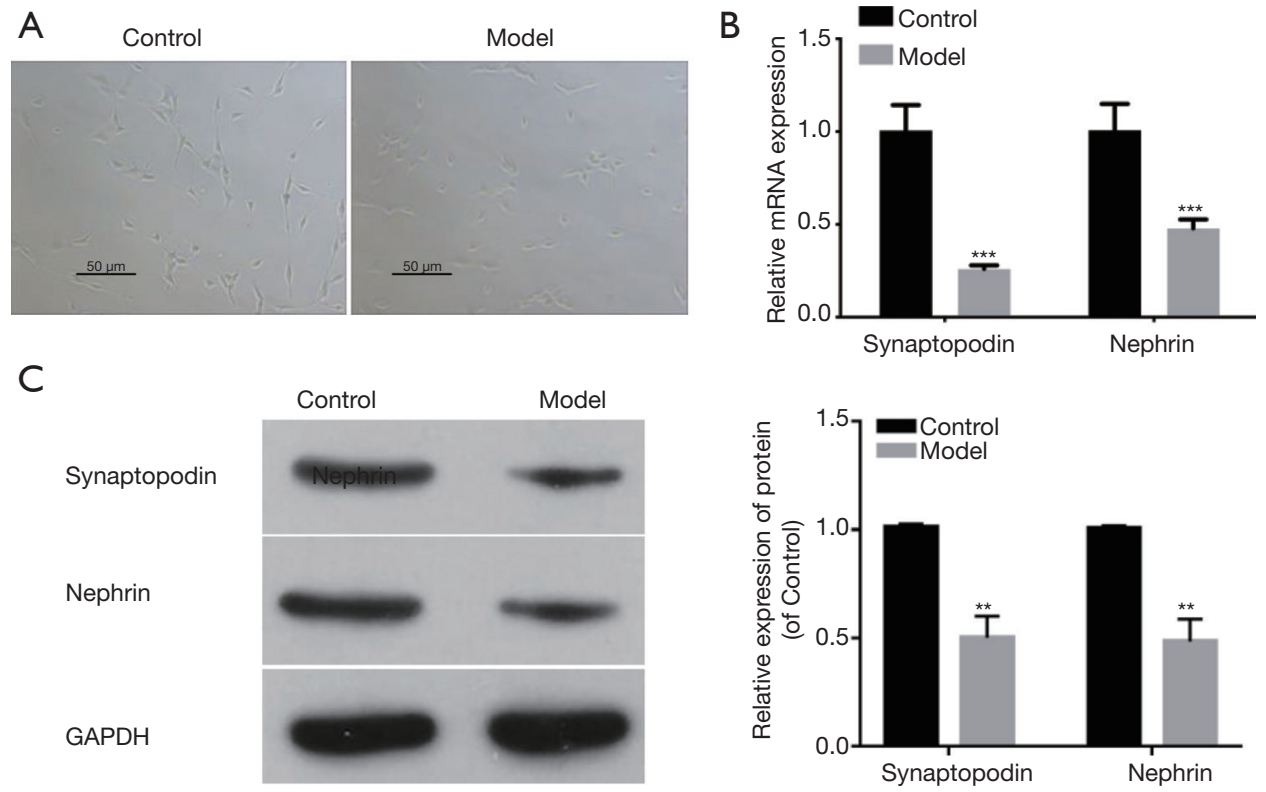

Figure 1 Podocyte injury in adriamycin (ADR)-treated MPC5 cells. (A) Effect of ADR treatment on MPC5 cell morphology. (B,C) Quantitative real-time PCR and western blot were used to measure the mRNA and protein levels of synaptopodin and nephrin in control and model cells, respectively. The experimental data are shown as mean \pm standard deviation $(\mathrm{SD}) . \mathrm{N}=3 .{ }^{* *}$ represents $\mathrm{P}<0.01 ;{ }^{* * *}$ represents $\mathrm{P}<0.001$. Magnification $100 \times$, scale bar $=50 \mu \mathrm{m}$.

diacetate (DCF-DA) for 30 minutes in the dark at $37^{\circ} \mathrm{C}$, and the resulting DCF fluorescence was analyzed through flow cytometry.

\section{Hematoxylin and eosin (HE) staining}

HE staining was carried out as described in previous studies (12). Mouse renal tissues were placed in $10 \%$ neutral-buffered formalin fixative at room temperature overnight, embedded in paraffin, and stained with HE.

\section{Statistical analysis}

At least three biological replicates of each experiment were performed. All experimental data were expressed as means \pm standard deviations (SDs). Student's $t$-test was utilized to analyze differences between two groups, and one-way analysis of variance was performed to evaluate differences between 3 or more groups. Statistical analyses were performed with GraphPad (Ver. Prism 7, GraphPad Prism Software, La Jolla, CA, USA). A P value $<0.05$ was considered to be statistically significant.

\section{Results}

\section{Podocyte injury in ADR-treated MPC5 cells}

To develop the nephropathy cell model, MPC5 cells were treated with ADR $(100 \mu \mathrm{M})$ for 24 hours. Compared with the control group, cells in the model group showed significant injury (Figure $1 A$ ). The results of qRT-PCR and western blot further indicated that the mRNA and protein expression levels of the slit diaphragm molecules synaptopodin and nephrin were reduced in the model cells (Figure 1B,1C). These data demonstrated that ADR induced podocyte injury.

\section{ADR treatment inhibits podocyte proliferation while promoting apoptosis}

We then further investigated the effects of ADR on MPC5 cell proliferation and apoptosis. First, we examined the proliferation ability of treated cells by conducting CCK-8 and EdU assays. The results showed that ADR treatment significantly reduced MPC5 cell proliferation (Figure 2A,2B). Next, western blot was performed to 

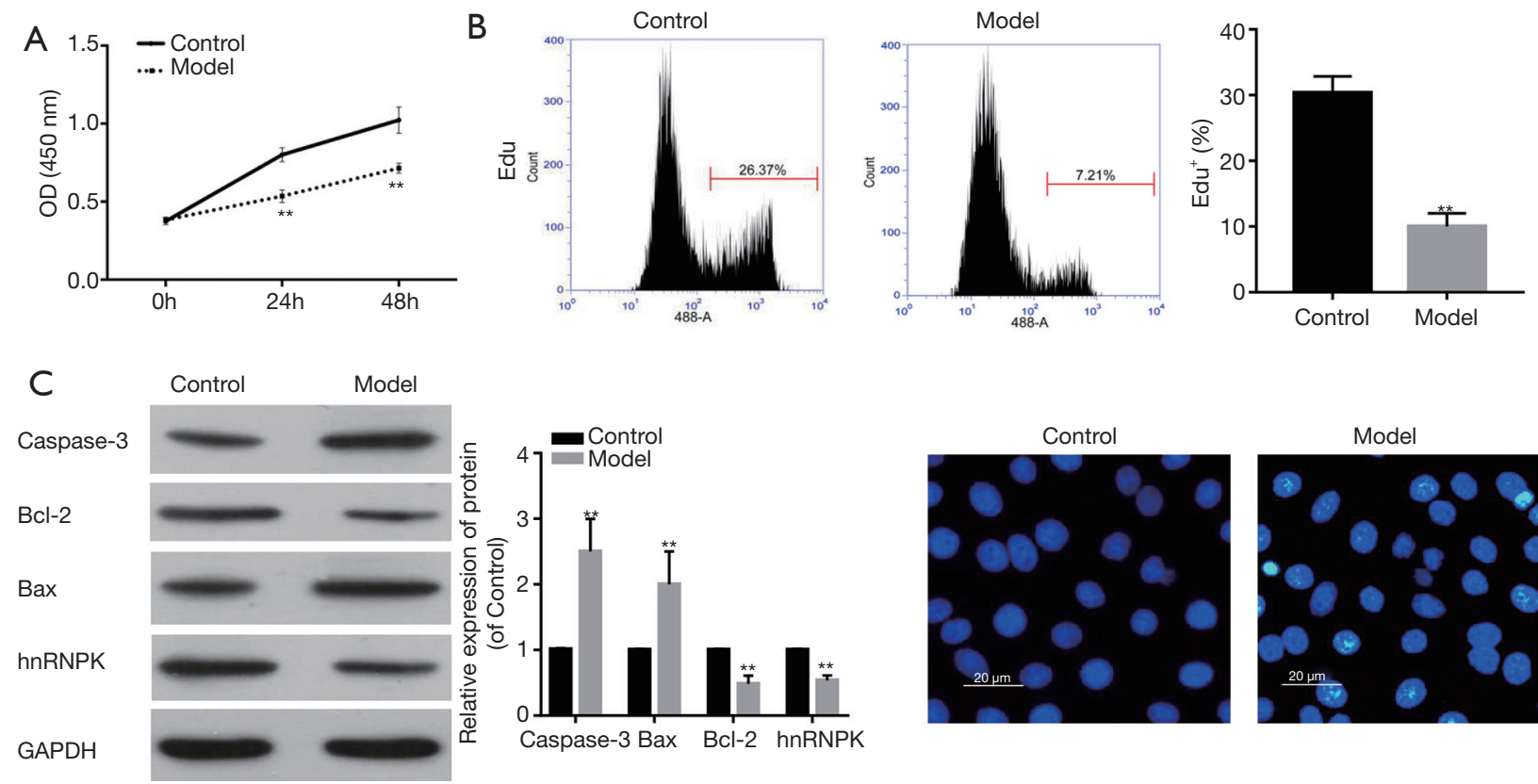

Figure 2 Adriamycin (ADR) treatment inhibits podocyte proliferation while promoting apoptosis. (A,B) Cell proliferation of treated MPC5 cells was analyzed using Cell Counting Kit-8 assay and EdU assay. (C) Expression levels of caspase-3, Bcl-2, Bax, and heterogeneous nucleotide protein K (hnRNPK) were detected by western blot, with GAPDH used as an internal control. (D) Hoechst staining was performed to examine cell apoptosis. The experimental data are shown as mean \pm standard deviation $(\mathrm{SD})$. $\mathrm{N}=3$. ** represents $\mathrm{P}<0.01$. Magnification $400 \times$, scale bar $=20 \mu \mathrm{m}$.

detect the expression of apoptotic proteins. The results showed that ADR increased caspase- 3 and Bax expression but decreased Bcl-2 expression (Figure 2C). Furthermore, hnRNPK protein expression was lower in the ADR-induced model group than in the control group (Figure 2C). Finally, to further analyze cell apoptosis, we used Hoechst 33258 to stain cell nuclei, and observed more apoptotic cells in the model group than in the control group (Figure 2D). Together, our results demonstrated that ADR reduced cell proliferation while increasing cell apoptosis.

\section{ADR inbibits bnRNPK expression in podocytes}

MMP disruption, LDH leakage, and ROS generation can also be used to evaluate cell injury. Firstly, as decreased MMP is a marker of early apoptosis, we conducted a JC-1 assay, and the results indicated a decrease in MMP in ADRtreated MPC5 cells (Figure $3 A$ ). The release of LDH can be used to evaluate cell membrane integrity, and excessive ROS can damage cells, so we performed experiments to examine both. Compared with the control group, ADR-treated cells showed significant increases in LDH concentration and ROS generation (Figure 3B,3C). These data further confirmed that ADR treatment could induce MPC5 cell injury.

\section{bnRNPK reverses the inbibitory effects of ADR treatment on podocytes}

Since hnRNPK expression was low in ADR-treated cells, we next sought to determine whether an elevated expression of hnRNPK could play a protective role in podocyte injury. Firstly, we evaluated the protein expression levels of hnRNPK and apoptotic proteins following treatment with ADR and hnRNPK. In the model + hnRNPK group, the expression levels of hnRNPK and Bcl-2 were increased, while those of caspase- 3 and Bax were decreased, compared to the model + vector group (Figure 4A). Next, the results from CCK-8 assay and EdU assay indicated that hnRNPK, to some extent, reversed the inhibitive effect of ADR treatment on MPC5 cell proliferation (Figure 4B,4C). Moreover, the Hoechst 33258 staining results also demonstrated that hnRNPK inhibited the cell apoptosis induced by ADR treatment (Figure 4D). These findings 

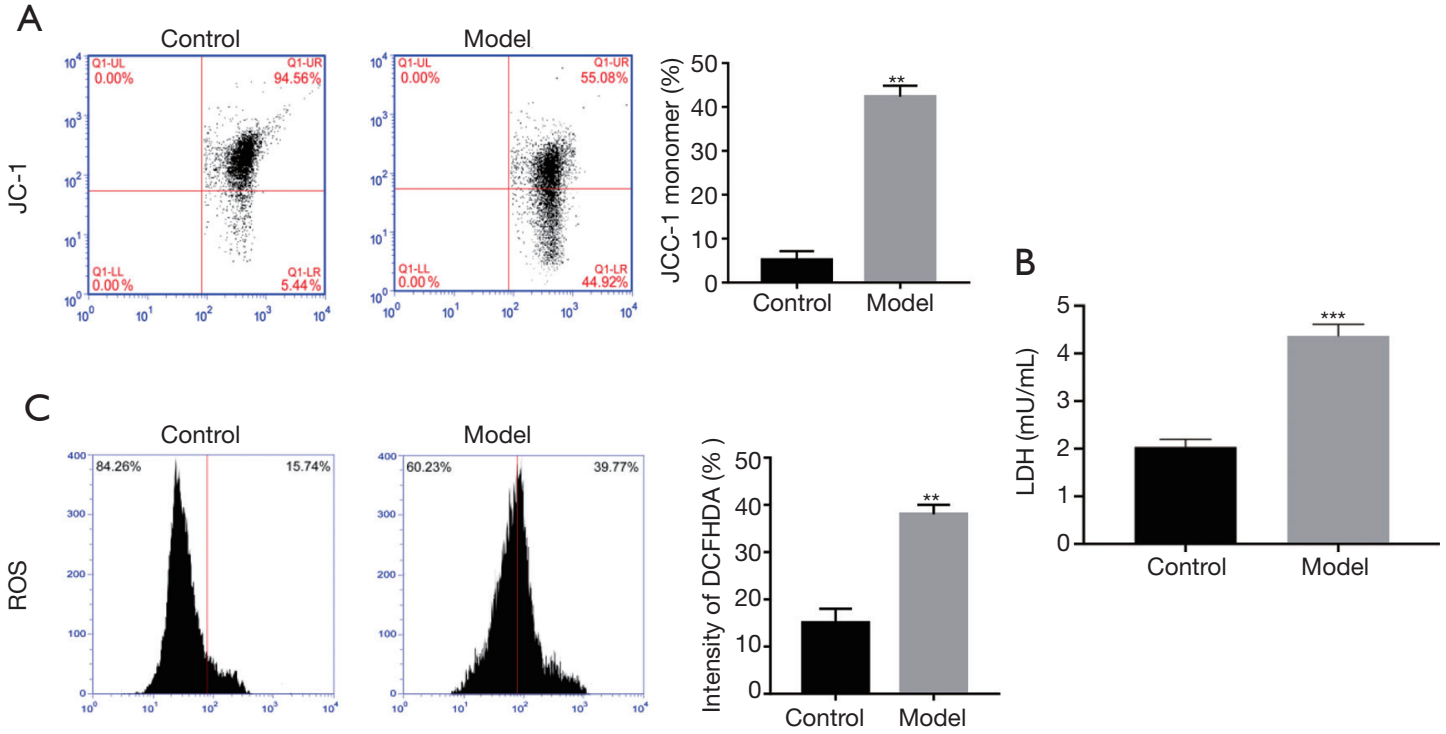

Figure 3 Adriamycin (ADR) elevates the mitochondrial membrane potential (MMP), reactive oxygen species (ROS), and lactate dehydrogenase $(\mathrm{LDH})$ in podocytes. (A) The MMP of treated MPC5 cells was measured by JC-1 assay. (B,C) Effects of ADR treatment on LDH leakage and ROS level in MPC5 cells. The experimental data are shown as mean \pm standard deviation (SD). N=3. ** represents $\mathrm{P}<0.01 ;{ }^{* * *}$ represents $\mathrm{P}<0.001$.

suggest that hnRNPK can protect against ADR-induced podocyte injury.

\section{bnRNPK attenuates ADR-induced MMP disruption, ROS generation, and LDH leakage in podocytes}

To further confirm the protective effects of hnRNPK on ADR-treated cells, we next evaluated MMP, LDH leakage, and ROS generation in treated MPC5 cells. As shown in Figure $5 A$, hnRNPK treatment inhibited the decrease in MMP in ADR-treated MPC5 cells. Furthermore, compared with the model + vector group, the model + hnRNPK group displayed significant decreases in ROS generation and LDH leakage (Figure 5B,5C). Taken together, our results suggested that hnRNPK reversed ADR-induced podocyte injury.

\section{bnRNPK has protective effects against ADR-induced podocyte injury in vivo}

To further explore whether hnRNPK can also exert protective effects in vivo, we constructed animal models. Male mice were injected with ADR or ADR + hnRNPK via the tail vein, and their glomeruli were obtained as tissue samples. Subsequently, we evaluated the expression of hnRNPK and apoptotic proteins. The results showed that the expression levels of hnRNPK and Bcl-2 were decreased, while those of caspase- 3 and Bax were increased, in the model animals, and these effects were eliminated by hnRNPK overexpression (Figure 6A). Furthermore, the $\mathrm{HE}$ staining results showed that compared with the control tissues, overexpression of hnRNPK improved tubular expansion or deformation in ADR-treated mice (Figure 6B), suggesting that hnRNPK reduces renal damage.

\section{Discussion}

Podocytes are an essential part of the glomerular filtration barrier. Their injury can lead to proteinuria, which is a recognized marker of renal damage and a sign of decreased kidney function (13-15). Therefore, protecting podocytes from injury may be a valuable therapeutic strategy in the treatment of proteinuria-associated renal disease. So far, several drugs have been found to treat podocyte injury. For instance, a previous study reported that olmesartan reduced proteinuria by inhibiting podocyte apoptosis (16). Another study demonstrated that sinomenine reduced podocyte autophagy, which plays a key role in preventing podocyte depletion (17). However, it is still necessary to investigate the specific mechanisms of podocyte injury and 


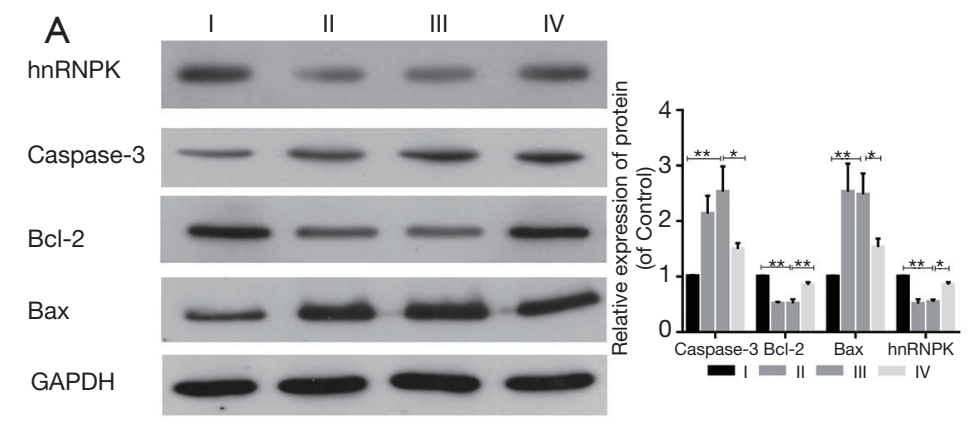

B

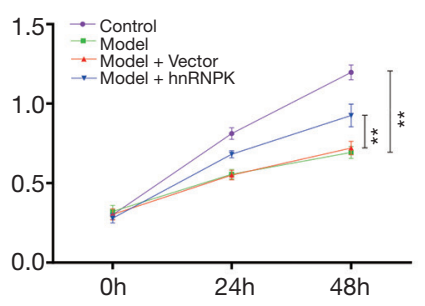

C

I: Control II: Model III: Model + Vector IV: Model + hnRNPK

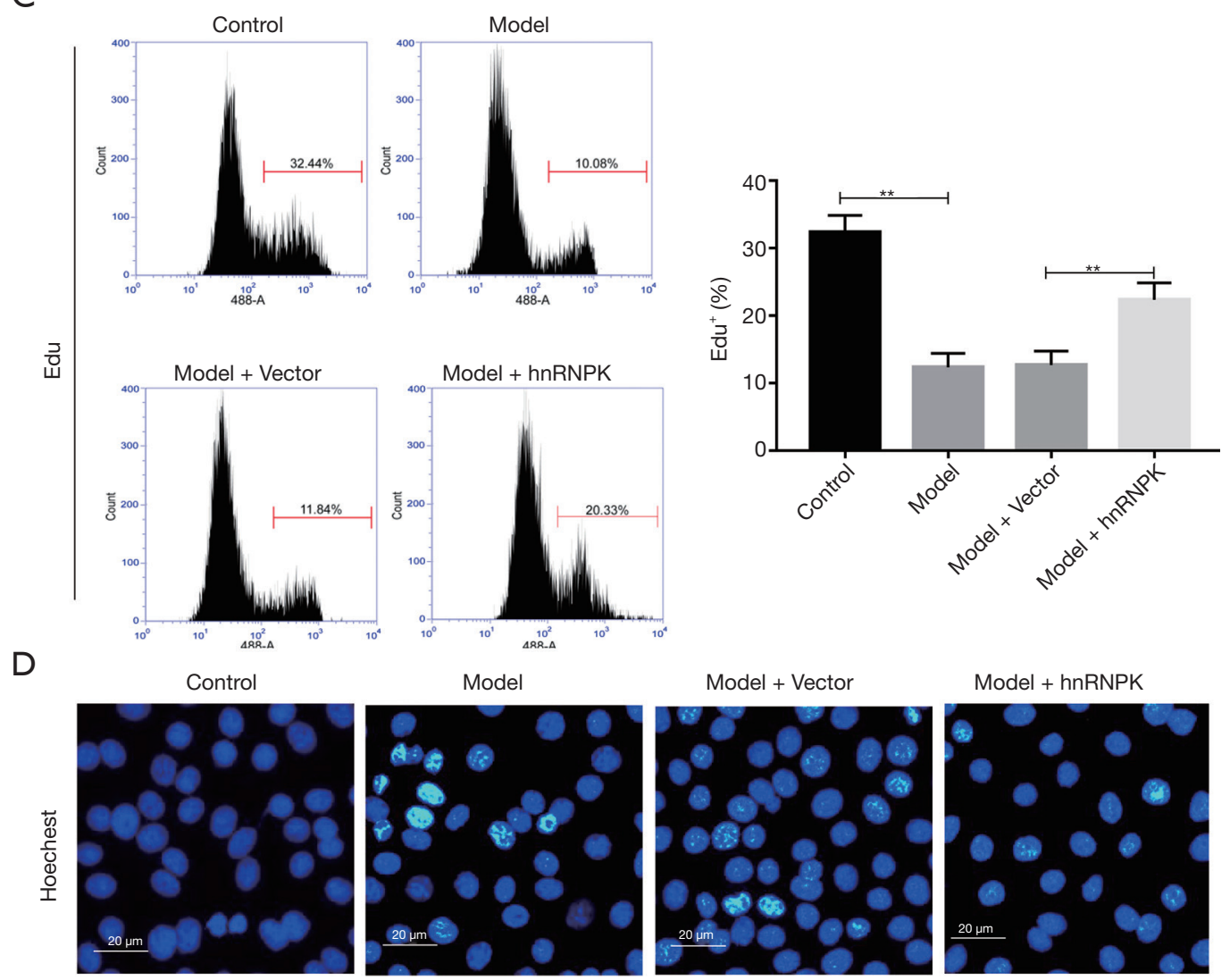

Figure 4 Heterogeneous nucleotide protein $\mathrm{K}$ (hnRNPK) reverses the inhibitory effects of adriamycin (ADR) treatment on podocytes. (A) Expression levels of hnRNPK, caspase-3, Bcl-2, and Bax in ADR-treated podocytes were measured by western blot after transfection with hnRNPK. (B-D) After transfection with hnRNPK, the cell proliferation of ADR-treated MPC5 cells were detected by Cell Counting Kit-8 assay, EdU assay, and the cell apoptosis of ADR-treated MPC5 cells were detected by Hoechst staining. The experimental data are shown as mean \pm standard deviation $(\mathrm{SD}) . \mathrm{N}=3$. * represents $\mathrm{P}<0.05$; ** represents $\mathrm{P}<0.01$. Magnification $400 \times$, scale bar $=20 \mu \mathrm{m}$.

to discover more effective drugs. In this study, we identified that hnRNPK was downregulated in podocytes with ADR-induced injury and that overexpression of hnRNPK alleviated ADR-related damage in vivo and in vitro, which suggests that hnRNPK might serve as a therapeutic agent for preventing podocyte injury.

ADR is a widely used anticancer drug $(18,19)$ which causes dose-dependent toxicity in the heart and kidneys; thus, its clinical application is limited (20). However, the ADR-induced podocyte injury model is mature, highly 

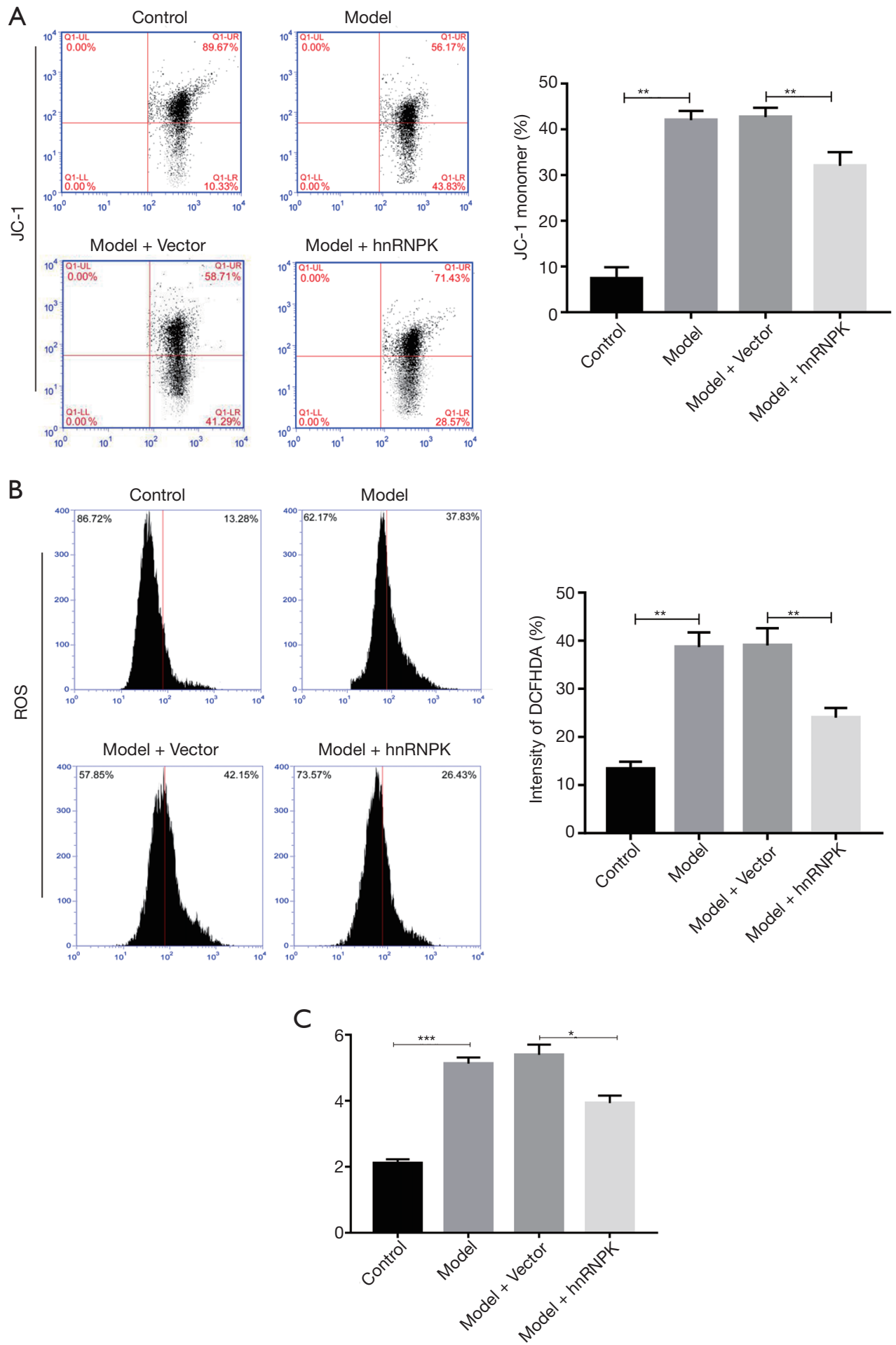

Figure 5 Heterogeneous nucleotide protein K (hnRNPK) attenuated adriamycin (ADR)-induced mitochondrial membrane potential (MMP) disruption, reactive oxygen species (ROS) generation, and lactate dehydrogenase (LDH) leakage in podocytes. (A) The MMP of ADRtreated podocytes was measured using JC-1 assay after transfection with hnRNPK. (B,C) Effects of hnRNPK overexpression on ROS level and LDH leakage in ADR-treated MPC5 cells. The experimental data are shown as mean \pm standard deviation $(\mathrm{SD}) . \mathrm{N}=3 .{ }^{*}$ represents $\mathrm{P}<0.05 ;{ }^{* *}$ represents $\mathrm{P}<0.01 ;{ }^{* * *}$ represents $\mathrm{P}<0.001$. 
A
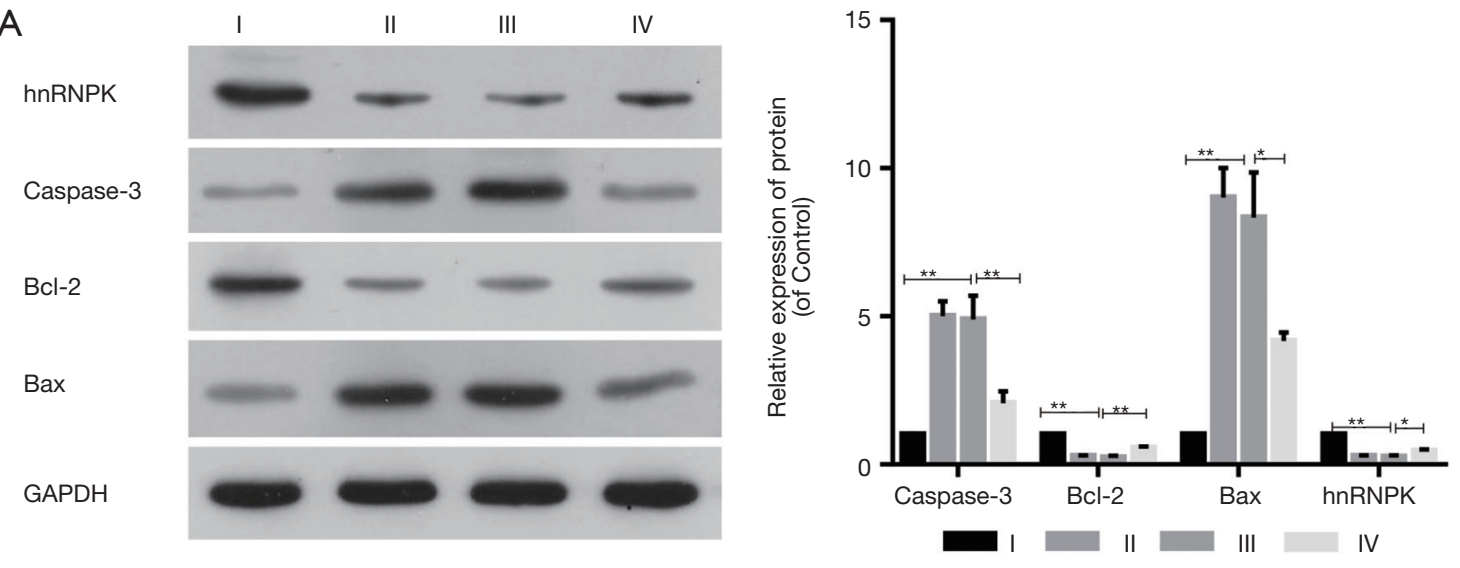

I: Control II: Model III: Model + Vector IV: Model + hnRNPK

B
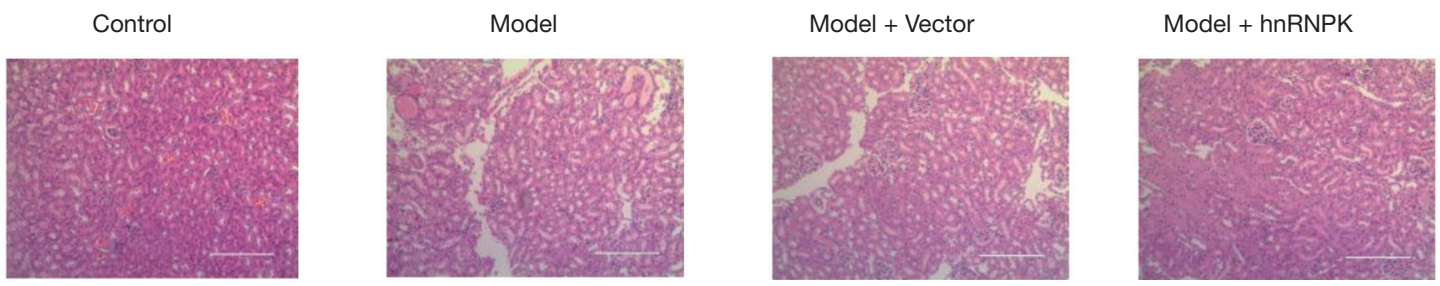

Figure 6 Heterogeneous nucleotide protein K (hnRNPK) has protective effects against adriamycin (ADR)-induced podocyte injury in vivo. (A) The expression levels of hnRNPK, caspase-3, Bcl-2, and Bax in glomeruli tissues from the different groups were measured using western blot. (B) The pathological changes in mouse renal tissues were analyzed by hematoxylin and eosin staining. Magnification $200 \times$, scale bar $=50 \mu \mathrm{m}$. The experimental data are shown as mean \pm standard deviation $(\mathrm{SD}) . \mathrm{N}=3 .{ }^{*}$ represents $\mathrm{P}<0.05,{ }^{* *}$ represents $\mathrm{P}<0.01$.

replicable, and widely used (21-23). Therefore, in the present study, $\mathrm{ADR}$ was used to establish a podocyte injury model. We first observed that the cytoskeleton structure of podocytes with ADR-induced injury had been damaged, and we then measured the expression of scaffold proteins, which play a key role in maintaining the integrity of the glomerular filtration barrier $(24,25)$. We also found that synaptopodin and nephrin were significantly decreased in ADR-injured podocytes.

Podocyte apoptosis refers to the characteristic phenomena of cell membrane vacuoles, nuclear condensation, and cell shrinkage under the influence of pathological factors (26). Podocyte apoptosis is one of the main reasons for decreases in podocyte number and density (7). Research has demonstrated that the Bcl-2 family and caspases have close relationships with cell apoptosis (27). The Bcl-2 family consists of 15 members, which can be divided into the Bcl2 subfamily, Bax subfamily, and Bel-2 homologous domainBH3 subfamily (28). The activation of caspases can also lead to the cleavage of related proteins, which is a key link in cell apoptosis (29); among them, caspase-3 is the main apoptotic effector protein (30). In the present study, we further verified that ADR significantly inhibited the proliferation of and induced the apoptosis of podocytes. Additionally, our results prove that ADR can upregulate caspase- 3 and Bax, and downregulate Bcl-2 in podocytes, which further suggests that ADR-induced podocyte injury induces apoptosis. Moreover, we discovered that hnRNPK expression was low in ADR-induced podocytes and that hnRNPK had a protective effect on podocyte apoptosis treated with ADR. Our findings indicate that compared with injured podocytes, podocytes co-treated with hnRNPK exhibited increased proliferation and their apoptosis was suppressed, suggesting that hnRNPK, to a certain extent, protects podocytes from ADR-induced injury.

To further verify the protective effect of hnRNPK against ADR-induced injury, we next measured MMP disruption, ROS generation, and LDH leakage in different groups. Mitochondria are the main source of ROS generation in cells (31), and mitochondrial dysfunction can 
lead to excessive ROS generation and accumulation (32). Increasing evidence shows that a moderate amount of ROS is beneficial to cell proliferation and differentiation, whereas excessive ROS can cause oxidative stress, leading to cell damage and apoptosis $(33,34)$. Oxidative stress plays a key role in the mediation of podocyte injury and proteinuria (35). Excessive ROS synthesis and mitochondrial dysfunction are the main causes of diabetic nephropathy (36). Furthermore, as a soluble cytoplasmic enzyme, $\mathrm{LDH}$ is released into the extracellular space when the plasma membrane is damaged, so LDH leakage serves as an indicator of cell damage (37). Our experimental results show that ROS generation and LDH leakage were significantly increased in podocytes with ADR-induced injury, whereas overexpression of hnRNPK reduced the levels of ROS and LDH, thus further demonstrating that hnRNPK can alleviate ADRinduced podocyte injury. Moreover, in vivo experiments were performed to detect the expression of hnRNPK and apoptotic proteins in mouse tissues. The results showed that hnRNPK and Bcl-2 were downregulated, while caspase- 3 and Bax were upregulated, in podocyte-injured mice. The opposite results were observed in tissues with hnRNPK pretreatment. Subsequently, the results of HE staining indicated histological changes in the ADR-treated mice, as evidenced by nuclear irregularity and karyorrhexis. However, these defects were ameliorated by pretreatment with hnRNPK. Although hnRNPK has been shown to protect against ADR-induced podocyte injury both in vitro and in vivo, the mechanism underlying its protective effect is still unknown and will therefore be the focus of our next study.

In conclusion, our findings suggest that hnRNPK can ameliorate ADR-induced podocyte injury both in vitro and in vivo. This observation provides a basis for a feasible therapy to prevent podocyte injury and subsequent kidney disease.

\section{Acknowledgments}

Funding: This work was supported by Health Science and Technology Project of Guangzhou (grant no. 20201A011115), Traditional Chinese Medicine \& Integrated Chinese and Western Medicines Science and Technology Project of Guangzhou (grant no. 20202A010023), Science and Technology Planning Project of Guangzhou City Huadu District (grant no. 19-HDWS-033), the Scientific Research Foundation of Huadu District People's Hospital of Guangzhou (grant no. 2019B01) and Guangzhou Key
Discipline of Medicine (2021-2023).

\section{Footnote}

Reporting Checklist: The authors have completed the ARRIVE reporting checklist. Available at https://dx.doi. org/10.21037/atm-21-3577

Data Sharing Statement: Available at https://dx.doi. org/10.21037/atm-21-3577

Conflicts of Interest: All authors have completed the ICMJE uniform disclosure form (available at https://dx.doi. org/10.21037/atm-21-3577). The authors have no conflicts of interest to declare.

Ethical Statement: The authors are accountable for all aspects of the work in ensuring that questions related to the accuracy or integrity of any part of the work are appropriately investigated and resolved. All experiments were performed and approved by the Affiliated Huadu Hospital, Southern Medical University, in compliance with the Affiliated Huadu Hospital of Southern Medical University guidelines for the care and use of animals.

Open Access Statement: This is an Open Access article distributed in accordance with the Creative Commons Attribution-NonCommercial-NoDerivs 4.0 International License (CC BY-NC-ND 4.0), which permits the noncommercial replication and distribution of the article with the strict proviso that no changes or edits are made and the original work is properly cited (including links to both the formal publication through the relevant DOI and the license). See: https://creativecommons.org/licenses/by-nc-nd/4.0/.

\section{References}

1. Yu J, Gong $\mathrm{W}, \mathrm{Wu} \mathrm{Y}$, et al. mPGES-1-derived PGE2 contributes to adriamycin-induced podocyte injury. Am J Physiol Renal Physiol 2016;310:F492-8.

2. Chen X, Qin Y, Zhou T, et al. The potential role of retinoic acid receptor $\alpha$ on glomerulosclerosis in rats and podocytes injury is associated with the induction of MMP2 and MMP9. Acta Biochim Biophys Sin (Shanghai) 2017;49:669-79.

3. Lu CC, Wang GH, Lu J, et al. Role of Podocyte Injury in Glomerulosclerosis. Adv Exp Med Biol 2019;1165:195-232.

4. Pavenstädt H, Kriz W, Kretzler M. Cell biology of the 
glomerular podocyte. Physiol Rev 2003;83:253-307.

5. Welsh GI, Saleem MA. The podocyte cytoskeleton--key to a functioning glomerulus in health and disease. Nat Rev Nephrol 2011;8:14-21.

6. Mathieson PW. The podocyte as a target for therapies-new and old. Nat Rev Nephrol 2011;8:52-6.

7. Nagata M. Podocyte injury and its consequences. Kidney Int 2016;89:1221-30.

8. Fan X, Xiong H, Wei J, et al. Cytoplasmic hnRNPK interacts with GSK $3 \beta$ and is essential for the osteoclast differentiation. Sci Rep 2015;5:17732.

9. Wang Z, Qiu H, He J, et al. The emerging roles of hnRNPK. J Cell Physiol 2020;235:1995-2008.

10. Gallardo M, Lee HJ, Zhang X, et al. hnRNP K Is a Haploinsufficient Tumor Suppressor that Regulates Proliferation and Differentiation Programs in Hematologic Malignancies. Cancer Cell 2015;28:486-99.

11. Abdo S, Lo CS, Chenier I, et al. Heterogeneous nuclear ribonucleoproteins $\mathrm{F}$ and $\mathrm{K}$ mediate insulin inhibition of renal angiotensinogen gene expression and prevention of hypertension and kidney injury in diabetic mice. Diabetologia 2013;56:1649-60.

12. Long Y, Yao DS, Wei YS, et al. Perineural invasion in early-stage cervical cancer: detection and influence on prognosis. European Journal of Gynaecological Oncology 2019;40:452-6.

13. Jafar TH, Stark PC, Schmid CH, et al. Proteinuria as a modifiable risk factor for the progression of non-diabetic renal disease. Kidney Int 2001;60:1131-40.

14. Turin TC, James M, Ravani P, et al. Proteinuria and rate of change in kidney function in a community-based population. J Am Soc Nephrol 2013;24:1661-7.

15. Azuma K, Suzuki S, Ishii Y, et al. Tortuosity of the brachiocephalic artery complicated with arterial injury after tracheotomy: a case report. Signa 2019;15:77-8.

16. Gu J, Yang M, Qi N, et al. Olmesartan Prevents Microalbuminuria in $\mathrm{db} / \mathrm{db}$ Diabetic Mice Through Inhibition of Angiotensin II/p38/SIRT1-Induced Podocyte Apoptosis. Kidney Blood Press Res 2016;41:848-64.

17. Wang W, Cai J, Tang S, et al. Sinomenine Attenuates Angiotensin II-Induced Autophagy via Inhibition of P47Phox Translocation to the Membrane and Influences Reactive Oxygen Species Generation in Podocytes. Kidney Blood Press Res 2016;41:158-67.

18. Chen C, Lu L, Yan S, et al. Autophagy and doxorubicin resistance in cancer. Anticancer Drugs 2018;29:1-9.

19. Chen Z, An X, Liu X, et al. Hyperoside alleviates adriamycin-induced podocyte injury via inhibiting mitochondrial fission. Oncotarget 2017;8:88792-803.

20. Hou XW, Jiang Y, Wang LF, et al. Protective role of granulocyte colony-stimulating factor against adriamycin induced cardiac, renal and hepatic toxicities. Toxicol Lett 2009;187:40-4.

21. Pippin JW, Brinkkoetter PT, Cormack-Aboud FC, et al. Inducible rodent models of acquired podocyte diseases. Am J Physiol Renal Physiol 2009;296:F213-29.

22. Susztak K, Raff AC, Schiffer M, et al. Glucose-induced reactive oxygen species cause apoptosis of podocytes and podocyte depletion at the onset of diabetic nephropathy. Diabetes 2006;55:225-33.

23. Saito Y, Okamura M, Nakajima S, et al. Suppression of nephrin expression by TNF-alpha via interfering with the cAMP-retinoic acid receptor pathway. Am J Physiol Renal Physiol 2010;298:F1436-44.

24. Empitu MA, Kadariswantiningsih IN, Aizawa M, et al. MAGI-2 and scaffold proteins in glomerulopathy. Am J Physiol Renal Physiol 2018;315:F1336-44.

25. Kato T, Mizuno S. Nephron, Wilms' tumor-1 (WT1), and synaptopodin expression in developing podocytes of mice. Exp Anim 2017;66:183-9.

26. Yu S, Ren Q, Yu L, et al. Role of autophagy in Puromycin Aminonucleoside-induced podocyte apoptosis. J Recept Signal Transduct Res 2020;40:273-80.

27. Voss AK, Strasser A. The essentials of developmental apoptosis. F1000Res 2020;9:F1000 Faculty Rev-148.

28. Kale J, Osterlund EJ, Andrews DW. BCL-2 family proteins: changing partners in the dance towards death. Cell Death Differ 2018;25:65-80.

29. Espinosa-Oliva AM, García-Revilla J, Alonso-Bellido IM, et al. Brainiac Caspases: Beyond the Wall of Apoptosis. Front Cell Neurosci 2019;13:500.

30. Julien O, Wells JA. Caspases and their substrates. Cell Death Differ 2017;24:1380-9.

31. Mammucari C, Rizzuto R. Signaling pathways in mitochondrial dysfunction and aging. Mech Ageing Dev 2010;131:536-43.

32. Yuan $Y$, Huang $S$, Wang $W$, et al. Activation of peroxisome proliferator-activated receptor- $\gamma$ coactivator $1 \alpha$ ameliorates mitochondrial dysfunction and protects podocytes from aldosterone-induced injury. Kidney Int 2012;82:771-89.

33. Circu ML, Aw TY. Reactive oxygen species, cellular redox systems, and apoptosis. Free Radic Biol Med 2010;48:749-62.

34. Perry G, Raina AK, Nunomura A, et al. How important is oxidative damage? Lessons from Alzheimer's disease. Free Radic Biol Med 2000;28:831-4. 
35. Liu X, Cao W, Qi J, et al. Leonurine ameliorates adriamycin-induced podocyte injury via suppression of oxidative stress. Free Radic Res 2018;52:952-60.

36. Higgins GC, Coughlan MT. Mitochondrial dysfunction and mitophagy: the beginning and end to diabetic nephropathy? Br J Pharmacol 2014;171:1917-42.

Cite this article as: Zhao S, Feng J, Li J, Cao R, Zhang Y, Yang S, Yin L. The RNA binding protein hnRNPK protects against adriamycin-induced podocyte injury. Ann Transl Med 2021;9(16):1303. doi: 10.21037/atm-21-3577
37. Burd JF, Usategui-Gomez M. A colorimetric assay for serum lactate dehydrogenase. Clin Chim Acta $1973 ; 46: 223-7$.

(English Language Editors: A. Muijlwijk and J. Reynolds) 